\title{
LA EDUCACIÓN DESDE LAS VOCES DE LOS Y LAS ADOLESCENTES $^{1}$
}

\author{
EDUCATION AS SEEN BY YOUNG VOICES ${ }^{2}$
}

Rosa Amelia Vallejos Lizárraga ${ }^{3}$

\section{Resumen}

La educación es un derecho fundamental reconocido en la Constitución y en las normas nacionales e internacionales. Sin embargo, para esta ponencia, se resaltará la educación vista principalmente desde la escuela, al constituirse en uno de los espacios en los que los niños, niñas y adolescentes se desenvuelven a lo largo de catorce años de vida.

El fundamento de la educación desde las voces adolescentes toma como base los resultados de la Encuesta Young Voice Perú (voces adolescentes) aplicada por Save the Children Perú y el Informe Young Voice Perú 2017.

Se parte por reconocer la importancia del conocimiento de la Convención sobre los Derechos del Niño y en ese sentido, de sus derechos. La encuesta nos muestra que casi la mitad de los adolescentes nunca fueron informados sobre la Convención y que para variar, existe una confusión sobre el contenido de los mismos.

En esta revisión del tema es importante no perder de vista que los adolescentes perciben el valor de la educación desde su relación con la escuela y, al ser la educación un derecho, esta no se debe vulnerar a través de prácticas que no tomen en cuenta la opinión de los y las estudiantes más aún si en este espacio no se siente seguro y parte de sus preocupaciones fundamentales es cumplir con los deberes escolares que a veces no entienden ni comprenden sumado a que no reciben ayuda en casa.

\footnotetext{
Ponencia presentada en el Pre Congreso Mundial de los Derechos de la Niñez y la Adolescencia organizado por el Colegio de Abogados de Lima realizado el 7 y 8 de Setiembre de 2018 y que toma como referencia el Informe Young Voice Perú 2017 de Save the Children.

2 Paper presented at the Pre-World Congress on the Rights of Children and Adolescents organized by the Lima Bar Association realized on September $7^{\text {th }}-8^{\text {th }}, 2018$ and based on the Save the Children's 2017 Young Voice Peru Report. Abogada de la Universidad San Martin de Porres (UPSMP), Magister en Derecho Civil con Mención en Derechos de Familia de la Universidad Femenina del Sagrado Corazón (UNIFE). Docente en Diplomados de Derecho de Familia y Derechos del Niño, Docente de la Maestría de Sistemas de Protección de los Derechos del Niño, Niña y Adolescente contra la Violencia de la Universidad Nacional Herminio Valdizán, Miembro de la Comisión Consultiva de Familia, Niño, Niña y Adolescente y Coordinadora de Programas de Save the Children Perú.
} 
Palabras clave: Convención, escuela, voces adolescentes, participación y preocupaciones.

\begin{abstract}
:
Education is a fundamental right recognized in the Constitution and in national and international regulations. However, this paper mainly considers elementary and high schools, as it is one of the spaces where children and adolescents grow throughout fourteen years of their lives.
\end{abstract}

From adolescent voices' perspective, the foundation of education is based on the results of the Young Voice Peru Survey (adolescent voices) applied by Save the Children Peru and the Young Voice Peru 2017 Report.

The starting point is to recognize the importance of raising awareness on the Convention on the Rights of the Child and, in that sense, their rights. The survey shows that almost half of the adolescents has never heard about the Convention and, on top of that, there is confusion about its content.

In this review of the subject, it is important not to lose sight of the fact that adolescents attach value to education on the basis of their relationship with their school. Since education is a right, it should not be violated through practices that do not take into account students' opinions, especially if they do not feel safe in this environment or where part of their fundamental concerns is to comply with school assignments that they sometimes do not understand or have no help at home.

Keywords : Convention, School, Young Voices, Participation and Concerns.

\title{
INTRODUCCIÓN
}

El presente trabajo se preparó para la ponencia del Pre Congreso Mundial por los Derechos de la niñez y la adolescencia organizado por la Comisión de Familia, Niño, Niña y Adolescente del Colegio de Abogados de Lima.

En este sentido, desde Save the Children hemos realizado con esmero esta iniciativa del Young Voice (Voces adolescentes) con la finalidad de impulsar la participación activa de los adolescentes en las decisiones que afectan sus vidas como el conocimiento de sus derechos, las oportunidades que tienen para hacer oír su voz, la seguridad frente a la violencia y cualquier tipo de discriminación, la forma como perciben la escuela y la educación que reciben. Para esta ocasión, hemos considerado desarrollar dos de estas 
temáticas del informe, por lo que le hemos denominado: La educación desde las Voces adolescentes.

Para este efecto, se presenta los resultados que se pueden visualizar en el video Young Voice ${ }^{4}$ y luego se podrán apreciar en adelante todo ello con la finalidad de llevarnos a todos los adultos a reflexionar sobre cuánto estamos cumpliendo nuestro rol frente a nuestra niñez y adolescencia, y a tomar medidas urgentes para generar los cambios necesarios que permitan establecer nuevos parámetros sociales basados en la escucha activa, el respeto a los derechos y la tolerancia. Como podemos apreciar, Young Voice Perú nos muestra que aún queda mucho por hacer.

El valor de la educación en el ser humano es incalculable. Para las niñas, niños y adolescentes, el proceso educativo se da en la escuela y para muchos de ellos son sinónimos. El tipo de relación que tienen los adolescentes con la escuela (entendido no solo como espacio físico sino como sistema) influirá en el valor y el lugar que le den a la educación en sus vidas.

Conscientes de que la realidad de la educación es compleja y profunda, la encuesta Young Voice abordó algunos puntos críticos de esta realidad en la vida del adolescente, que serán de gran utilidad para generar estrategias de mejora.

Finalmente, les invitamos a apropiarse de este documento y a quitarse por un momento los lentes de adulto para mirar como niño, niña o adolescente. Busquemos ser innovadores, entender sus motivaciones, acoger sus necesidades y actuar para lograr los cambios que se requieren.

\section{YOUNG VOICE PERÚ - 2017}

Young Voice Perú es una encuesta realizada a nivel nacional en la que participaron adolescentes de todas las regiones del país. A través de esta iniciativa se busca conocer la opinión y puntos de vista de adolescentes acerca de las cuestiones que les afectan en aquellos escenarios donde se desarrollan, como la escuela, la ciudad, la casa o internet, y realidades con las que conviven, como la seguridad o inseguridad, la participación en la toma de decisiones, las responsabilidades escolares, sus derechos y deberes, su presente y su futuro, entre otros.

Link del video Young Voice Perú (2017) Disponible en: https://resourcecentre.savethechildren.net/library/ young-voices-peru-2017 
Como antecedente, la encuesta fue desarrollada originalmente por Save the Children Suecia en 2014. Los resultados se presentaron ante políticos suecos, formuladores de políticas públicas y proveedores de servicios y tuvieron un importante impacto. A partir de dicha experiencia, se ha impulsado la aplicación de la encuesta en Kosovo, Lituania, Mongolia, Armenia y Jordania.

Por su parte, el Perú es el primer país latinoamericano en poner en práctica dicha estrategia para contribuir a generar cambios importantes en nuestra sociedad y dejar un valioso precedente a otros países de la región.

Save the Children Perú decidió llevar a cabo la encuesta Young Voice para contribuir con la promoción y defensa de los derechos de los y las adolescentes. Esto a la luz de la Convención sobre los Derechos del Niño (CDN), que reconoce en su artículo 12, el derecho de todos los niños, niñas y adolescentes de expresar su opinión y ser escuchados. Y además, a ser tomados en serio, constituye uno de los valores fundamentales de la Convención.

\section{CONCIENCIA DE LOS DERECHOS Y DE LA CONVENCIÓN SOBRE LOS DERECHOS DEL NIÑO}

\section{"La libertad es un derecho para el adolescente... en la escuela nos hablan más sobre nuestros deberes" (Adolescente de Callao)}

La encuesta Young Voice Perú empieza explorando un aspecto fundamental de los y las adolescentes: la realidad sobre el conocimiento y reconocimiento de los derechos de los que son portadores. Los derechos de los adolescentes son un reflejo de su dignidad y valor. Frente a estos derechos, surgen las obligaciones del Estado, de los adultos y responsables, comenzando por la responsabilidad de "dar a conocer ampliamente los principios y disposiciones de la Convención por medios eficaces y apropiados, tanto a los adultos como a los niños", mandato recogido en el artículo $42^{\circ}$ de la Convención sobre los Derechos del Niño.

Los primeros resultados nos muestran que el $46.4 \%$ la mitad de los encuestados no ha recibido información sobre la Convención en el último año escolar, y un $37.7 \%$ no ha recibido nunca información al respecto.

Junto a estos valores, aparece otro igual de preocupante: un $16.3 \%$ de los encuestados no sabe si se expuso sobre la Convención en su escuela (en el último año), lo cual es muestra de un total desinterés de este nada desdeñable porcentaje de adolescentes. 


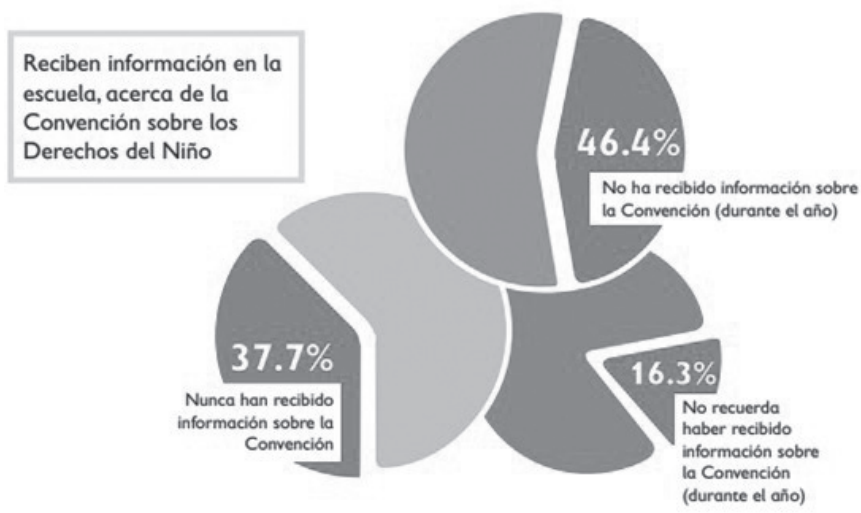

Figura 1. Encuesta Young Voice Perú.

Fuente: Young Voice Perú, 2017

Otro aspecto que llama la atención es el que los adolescentes reciben mayor información sobre sus derechos de manera general, y no dentro del contexto de la Convención. Solo un $37.4 \%$ de los encuestados ha recibido información sobre la Convención sobre los Derechos del niño durante el año escolar anterior, frente a un $51.4 \%$ que recibió información sobre sus derechos en general en el mismo lapso.

Durante los talleres con grupos focales, los adolescentes mostraron desconocimiento sobre la diferencia entre la información contenida en la Convención sobre los Derechos del Niño y los derechos del niño difundidos en un contexto general. Muchos de ellos se mostraban confundidos y señalaban que en el colegio les han enseñado que son 10 sus derechos, mientras que los que conocían sobre la Convención indicaban que habían recibido esta información en la organización (civil) a la que pertenecen.

\section{INFLUENCIA DE LA ESCUELA EN LA VIDA DEL ADOLESCENTE}

\section{"Yo conozco un colegio en la que te preparan para ser ama de casa"}

(Adolescente de Huancayo)

El valor de la educación en el ser humano es incalculable. Para las niñas, niños y adolescentes, el proceso educativo se da en la escuela y para muchos de ellos son sinónimos. El tipo de relación que tienen los adolescentes con la escuela (entendido no solo como espacio físico sino como sistema) influirá en el valor y el lugar que le den a la educación en sus vidas.

Conscientes de que la realidad de la educación es compleja y profunda, la encuesta Young Voice abordó algunos puntos críticos de esta realidad en la vida del adolescente, que serán de gran utilidad para generar estrategias de mejora. 
Respecto a las labores escolares que son las responsabilidades cotidianas que los adolescentes deben asumir. El 65.1\% de adolescentes señalaron que se sienten estresados siempre o a veces con las labores escolares. Pero ¿Cuáles podrían ser los motivos?

El 53.9\% comprende siempre o casi siempre las tareas que les dejan sus profesores, lo que significa que el otro $46.1 \%$ no las comprende o solo lo hace a veces.

Esta situación se vuelve aún más difícil cuando no reciben la ayuda necesaria para realizarlas. Más de la mitad (56.2\%) afirma no recibir, o solo a veces, ayuda en la escuela para realizar las tareas que la misma escuela les deja. Y en casa, el panorama se muestra muy similar: solo el $20.6 \%$ afirma recibir ayuda permanente en casa para cumplir con las tareas de la escuela, lo cual es bajo, frente a un $37.4 \%$ que solo a veces recibe ayuda o un $23.8 \%$ que nunca la recibe.

Es de esperar que estas condiciones generen un impacto considerable de angustia y preocupación en los adolescentes: el 62.2\% manifiesta haberse sentido preocupado durante el año por no poder cumplir con las tareas. Por último, el $44.1 \%$ se siente estresado debido a sus calificaciones.

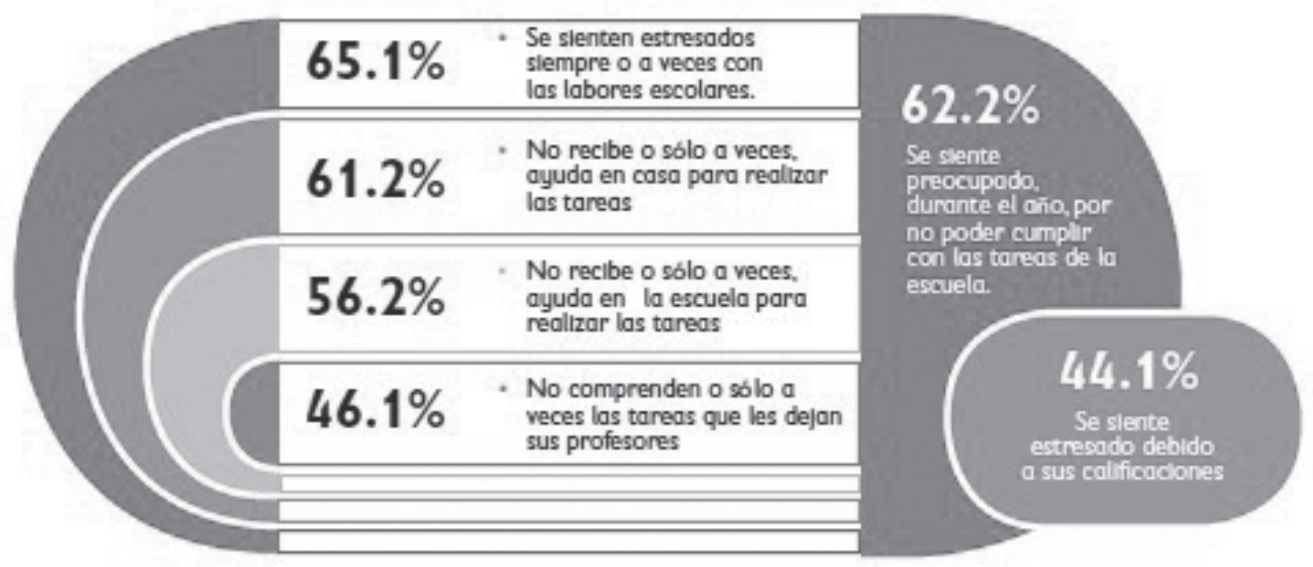

Figura 2. Encuesta Young Voice Perú sobre la realidad de la educación. Fuente: Young Voice Perú, 2017

En relación a la opinión del adolescente relacionado al tema de educación es el tema en el que mayor cantidad de adolescentes ha brindado opinión(10.5\%), pero a la vez es el tema en el que menos les gustaría brindar opinión (6\%). Solo el $8.9 \%$ ha podido dar opinión sobre asuntos que consideran importantes en 
la escuela. Sumado a que el 17.3\% ha participado en la elaboración del Plan de Convivencia Democrática en su escuela.

Complementariamente, el 42.3\% afirma que algún profesor (o alguien más en la escuela) le ha solicitado llevar dinero para participar en un paseo a realizarse fuera de la escuela. El 14.3\% ha dejado de participar de alguna actividad escolar porque ha costado dinero. A lo que se suma, que el $29.9 \%$ conoce del caso de una compañera de la escuela que ha dejado de estudiar por estar embarazada.

Al ser consultados sobre ciertos motivos que están detrás de la preocupación y el estrés por las labores escolares y las calificaciones, los adolescentes destacaron dos:

a) Existe más el miedo al juicio del adulto que a sentirse mal con ellos mismos por desaprobar.

En la casa, existe el temor de que los padres y madres se decepcionen de ellos o los castiguen. Se producen problemas familiares y hay distanciamiento entre padres, madres e hijos. El $67.3 \%$ no saben adónde ir cuando necesitan hablar con alguien o pedir ayuda si tienen problemas familiares.

En la escuela, hay las diferencias en el trato que reciben los estudiantes que desaprueban. Los adolescentes notaron cierta preferencia en el aula por los "más aplicados"; los estudiantes con las mejores notas representan al colegio y son más escuchados. Muchos profesores se muestran más intolerantes con los que desaprueban.

b) Existe temor a no tener un buen futuro al terminar el colegio si no sacan buenas notas.

\section{LA ESCUELA ME AYUDA}

El $25.3 \%$ se ha sentido preocupado el presente año por no recibir suficiente ayuda en la escuela. El hecho de que la cuarta parte de los encuestados tenga esta percepción de su institución educativa lleva a cuestionarnos el nivel de cercanía, promoción, protección o apoyo que la escuela debería tener con los estudiantes.

En los siguientes cuadros, se recopilan las opiniones de los adolescentes sobre muchos de estos temas: 

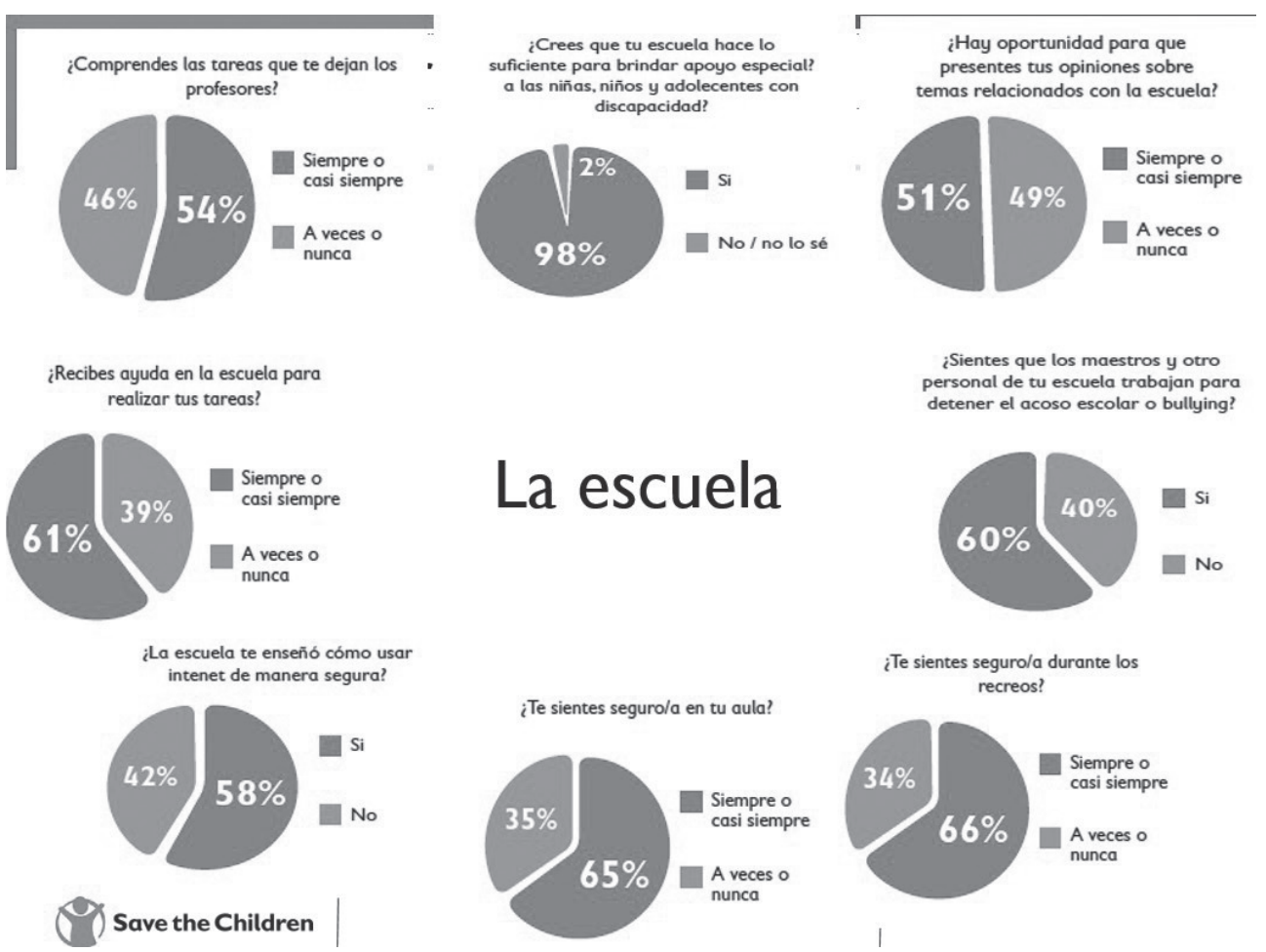

\section{La escuela}

¿Sientes que los maestros y otro personal de tu escuela trabajan para detener el acoso escolar o bullying?

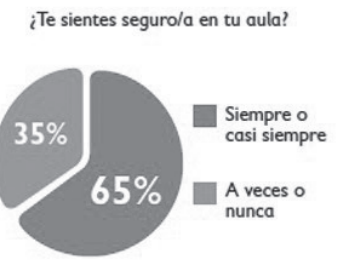

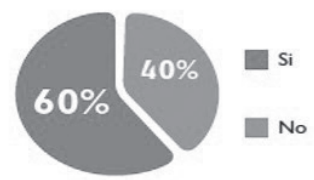

¿Te sientes seguro/a durante los recreos?

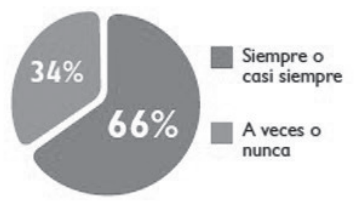

Figura 3. Temas sobre la escuela. Fuente: Save the Children, 2017

Además, hay un punto que preocupa de manera particular: el $28.3 \%$ se ha sentido preocupado durante el último año por la posibilidad de abandonar la escuela y no terminar los estudios. Si se contrasta esta información con las preocupaciones que tienen los adolescentes por la situación económica de su familia (32.2\%), el ser víctimas de bullying (22\%) o ser víctimas de violencia por parte de otros niños, niños o adolescentes (24.1\%), el haber huido de casa o haber sido expulsados de ella (25.2\%), el experimentar violencia por parte de un adulto $(21.4 \%)$, que no sepan a quién acudir si se sienten mal mentalmente $(53.1 \%)$ y un largo etcétera, se encontrarán muchas respuestas e insumos para que las autoridades puedan actuar.

Para concluir este tema, los adolescentes expresan su nivel de satisfacción sobre la enseñanza que reciben en la escuela. Esta pregunta va más allá de las materias o contenidos recibidos; cuestiona el nivel de pérdida-beneficio de asistir a la escuela con todo lo que esto trae consigo, y que hemos desarrollado líneas arriba. El 61.8\% se siente siempre (37.3\%) o casi siempre $(24.5 \%)$ satisfecho por la enseñanza recibida en la escuela, y el $38.2 \%$ a veces (26.15) o nunca satisfecho (12.1\%). Si tradujéramos esto en una nota o calificación, los adolescentes le habrían puesto un 12 a la enseñanza que reciben en su centro de estudios. 


\section{Nota de satisfacción respecto de la enseñanza}

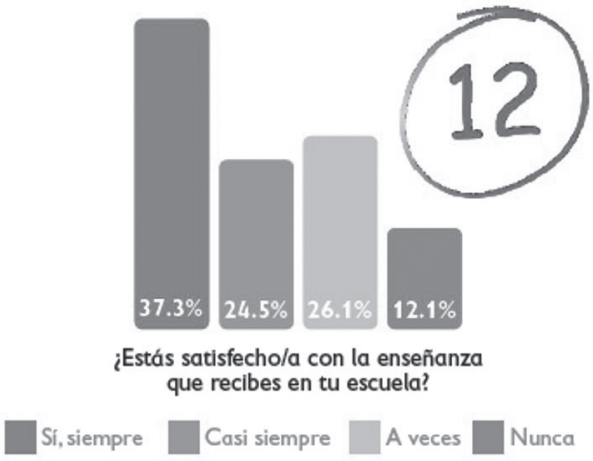

Figura 4. Nota de satisfacción respecto de la enseñanza.

Fuente: Young Voice Perú, 2017

Ahora bien, si vemos que al $99.4 \%$ no le gustaría dar opinión sobre educación en caso de tener la oportunidad de influir sobre sus autoridades, se entenderá que la nota que los adolescentes pondrían al binomio educación/ escuela sería abultadamente menor.

\section{CONCLUSIONES}

- Para garantizar el ejercicio de los derechos de niños, niñas y adolescentes es fundamental conocerlos. En el Perú, el $46.4 \%$ de los y las estudiantes adolescentes no han recibido información sobre la Convención sobre los Derechos del Niño en el último año escolar y un $37.7 \%$ no ha recibido nunca información al respecto. Siendo más grave aún que se haya podido comprobar que los que sí recibieron información sobre sus derechos no conozcan la diferencia entre los derechos contenidos en la Convención y los derechos del niño difundidos en un contexto general sumado a que en su mayoría señalaron que solo tenían diez derechos, clara referencia a la Declaración Universal de los Derechos del Niño.

- Unalabor pendientey urgente para el países asegurar elconocimientoy ejercicio de los derechos de los ciudadanos y ciudadanas, comenzando por los de los niños, niñas y adolescentes, jugando un rol importante en esta tarea los maestros y maestras, las autoridades educativas y el Estado que tiene la obligación de difundir la Convención.

- Es importante no perder de vista que los adolescentes perciben el valor de la educación desde su relación con la escuela y, al ser la educación un derecho, esta no se debe vulnerar a través de prácticas que no tomen en cuenta la opinión de los y las estudiantes. 
- La realidad mostrada a través del Informe Young Voice Perú genera preocupaciones toda vez que la tercera parte de estos no se sienten satisfechos con la enseñanza que reciben y el $6 \%$ prefiere no participar para brindar su opinión sobre la educación.

- Es necesario reevaluar el protagonismo que se le otorga a los deberes escolares y a las calificaciones, considerando el nivel de preocupación y estrés que generan en los estudiantes y el poco apoyo que se les brinda en la escuela para comprenderlos y cumplir con ellos.

- Debiendo considerarse que más de la cuarta parte de los estudiantes se ha sentido preocupado durante el último año por la posibilidad de abandonar la escuela y no terminar los estudios. Siendo que casi la tercera parte de estos, conoce del caso de una adolescente que ha dejado de estudiar por estar embarazada.

\section{REFERENCIAS}

MIMDES (2007) Convención sobre Derechos del Niño. Lima, Perú: Hilmart SA.

SAVE THE CHILDREN (2017) Informe Young Voice Perú 2017. Lima, Perú: Ediciones Nova Print SAC.

Fecha de recepción : : 28 de agosto de 2018

Fecha de aceptación : : 23 de octubre 2018 